\title{
ESTIMATING THE EFFECTS OF STRESS DURING OPERATIONAL CONDITIONS
}

\author{
Jon French \\ Human Factors and Systems Department \\ Embry Riddle Aeronautical University \\ Daytona Beach, FL 32114, U.S.A
}

\author{
Jeff Miller \\ Micro Analysis and Design \\ 11301 Corporate Way \\ Suite 101 \\ Orlando, FL 32817, U.S.A.
}

\begin{abstract}
The Physiological Stress Index (PSI) was developed to provide a rational means for estimating the physiological and behavioral impact of exposure to physical stressors, in this case extreme temperatures and fatigue related to insufficient sleep. Stress can be life threatening and certainly threatens mission effectiveness. It is often derived from combinations of sources such as overheating and lack of sleep, particularly on individuals involved in physically demanding activities such as shipboard firefighting. The PSI was used as a performance modifying adjunct to existing discrete event simulation models designed to estimate shipboard manning requirements given various operational scenarios. Additionally, the PSI was used to estimate the most effective work rest cycles and recovery time required before selected crewmembers could be returned to duties involving significant levels of physical exertion.
\end{abstract}

\section{INTRODUCTION}

The ability to realistically anticipate physiological stress is essential when conducting an analysis of teams operating in harsh environments so that optimum team size and composition can be determined. The Physiological Stress Index (PSI) was developed to provide a means for rapidly generating quantifiable and incremental physiological stress data for teams subjected to the combined influences of physical activity, fatigue and physical environment. The PSI makes use of the two existing algorithms, Thermal Stress Index (TSI) and the FAtigue DEgradation (FADE) equation, into the Total Crew Model (TCM) human performance modeling tool. The Total Crew Model is a discrete event modeling tool that simulates underway manning requirements for naval combatants from a macro analytical perspective to determine the adequacy of a proposed crew complement. The simulation considers the combined effects of crew size, skill levels, task assignments, work schedules, watch standing requirements, and operational scenario. The TSI (French et al. 2004) was de- veloped to provide a means for estimating the physiological severity of exposure to extreme temperatures and to provide a rational means for determining their most effective work rest firefighting cycles. A shipboard manning model utilizing the TSI equation may be used to determine the optimum number of personnel required to man teams involved in physically demanding tasks such as firefighting or shipboard flight deck operations. The TSI is also used to determine the recovery time teams require before they may be effectively used again and to predict the probability of individual crewmembers sustaining heat related casualties. The FADE functionality (Bowen et. al. 2003) was developed to provide a means for estimating the fatigue induced by the reduced and fragmented sleep typical of operational underway periods that can dramatically impair the effectiveness of a ship's crews. The TCM incorporates the FADE equation to predict the fatigue level for each crewmember for the duration of the simulation as they progress through their anticipated duties and evolutions. The FADE equation combines the individual circadian component with the linear awake degradation and parabolic sleep recovery components of inadequate sleep and duty cycles to predict ongoing fatigue levels (French, et. al. 2003). The impact of different crew configurations, watch rotation schedules and other manning concerns can be compared and evaluated with this approach to provide a means to select the least fatigue degrading solutions.

By combining the effects of TSI and FADE in the total crew model, the resulting PSI modeling algorithm will enable analysts and decision makers to determine the effectiveness of individuals or teams involved in performing cognitive or physically demanding tasks in a potentially hostile environment over the duration of an underway period given the combined effects of exhaustion and fatigue. Potential metrics used to determine effectiveness are task duration and error rate. Specifically, elevated levels of PSI result in increased task durations for physical and cognitive tasks. Additionally, the error rate associated with cognitive tasks increase with elevated levels of PSI. Within the 
TCM, these metrics may be used to dynamically control task duration times and error rates.

The psychophysiological consequences of fatigue induced by inadequate crew rest are of course very different from those of the thermal shock that might be experienced from firefighting. This paper does not attempt to equate them but rather, to estimate their factorial impact and to suggest the need to combine them to improve the realism of operational analysis. However, the impact of fatigue on homeostatic mechanisms, particularly autonomic responsiveness can be reasonably argued to dramatically weaken the physiological ability to stand and fight a shipboard fire. For example, the impact of sleep deprivation on sympathetic function is well known. Adrenergic desensitization occurs during sleep loss stress in military cadets (Opstad, Wiik, Haugen and Skrede 1994). Sleep loss degrades cardiovascular regulation (Ogawa, Kanbayashi, Satio et al. 2003). Even mild but chronic sleep debt can affect metabolic and endocrine responsiveness (Spiegel, Leproult and Van Cauter,1999) Thus, the ability of the firefighter's body to withstand the threats to homeostatic integrity is severely degraded by sleep deprivation.

\section{TOTAL CREW MODEL}

The basic total crew model simulates underway manning requirements for naval combatants from a macro perspective to determine the adequacy of a proposed crew complement. The TCM consists of a series of asynchronous (non-networked) tasks that represent every high-level task type performed by the crew. These include both work (e.g. watch, maintenance, administration, etc.) and non-work (e.g. sleep, eat, personal time, etc.) tasks and cover the entire twenty-four hour period for each crewmember, each day through the duration of the scenario. Crew assignments and mission scenario events are updated in the TCM every fifteen minutes. Typical scenarios are fourteen to twenty-eight days in length in order to capture the cumulative effects of fatigue. The simulation considers the combined effects of crew size, skill sets, work schedules, evolution task assignments, watch standing requirements, and operational scenario.

Simulation results are used to evaluate whether the assigned crew complement can successfully accomplish all underway operations and do so within acceptable time constraints and crew fatigue levels. Adjustments may be made to the manning hypotheses with the goal of optimizing the crew size and schedules. Subsequent iterations may be used to support recommendations for billet reductions or schedule modifications intended to improve crew performance over the course of the scenarios.

\section{THERMAL STRESS INDEX}

As a consequence of being warm blooded, the human body must maintain a fairly constant internal temperature within a narrow range, even though it is exposed to a wide variety of environmental temperature extremes. To keep internal body temperatures within safe limits, excess heat must be released or conserved, primarily through varying the rate and amount of blood circulating through the skin. These involuntary responses usually occur when the temperature of the blood departs from 97-99 $\mathrm{F}$ and are kept in balance and controlled by the central nervous system. During extreme heat exposure, with so much blood going to the external surface of the body for cooling, relatively less goes to the active muscles, the brain and other internal organs. As a result, physical endurance declines and mental fatigue occurs, leading to a reduction in stamina, alertness and mental acuity.

The Thermal Stress Index was developed to estimate safe exposure durations for firefighters onboard ship. It is intended to be used in discrete event simulation models to provide a means for estimating the physiological severity of exposure to extreme temperatures in order to improve the realism of human performance models and to provide a rational means for determining effective work rest cycles.

The TSI was developed from an estimate of the physical work produced in $\mathrm{Kcal} / \mathrm{minute}$, the ambient temperature of the surrounding environment and an adjustment for normal human basal metabolic rate (French, et al. 2004). The index also considers the effectiveness of insulation material worn by the individual and the extent of physiological reserves of energy that humans possess for rapid energy expenditure during work. The key components of the TSI are as shown in Figure 1.

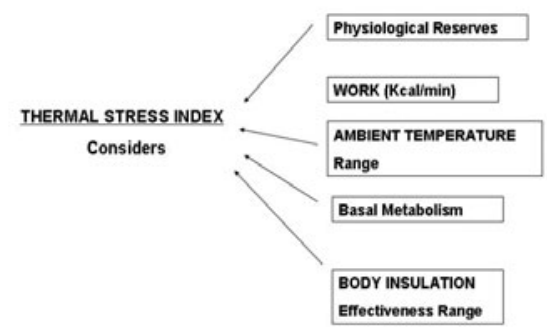

Figure 1. The Key Components of the TSI Equation.

Thermal stress is the sum of the heat generated by the body plus the heat gained from the environment minus the heat that is lost to the environment. The first term in the equation, the physiological reserve term, reflects the fact that we have a normal capacity to resist a certain amount of stress at the onset of heavy exertion in extreme temperatures before biological stress sets in. During exercise or heavy activity, muscles contribute a much higher percentage of the body's generated heat, hence the Work expendi- 
ture term. This heat must be dissipated to the environment to maintain normal body temperature. Ambient temperature is important because when working in hot environments, it becomes more difficult for the body to dissipate metabolic heat.

The Basal Metabolic heat term is that heat generated by the body even at rest. Firefighting crews wear protective equipment that allows them to survive in extreme heat. However, the suits are bulky and can be a source of increased metabolic heat from effort or work. The Insulation term in the equation provides ranges of protection but limits the savings in Kcal due to the added effort of movement. The Physiological Recovery term reflects the capacity to recover basal levels of temperatures with reduced workload and temperature and accounts for estimates of rest required to bring the TSI term to zero. It is convenient from the perspective of a rapid TSI estimate to use ranges rather than specific numbers. Hence, workload can be considered to be light ( $5 \mathrm{Kcal} /$ minute) or extremely heavy (15 Kcal/min) without knowing the exact Kcal/minute.

It has been established (McQuade 1975) that a persistent HSE value of $5 \mathrm{Kcal}$ per minute for about 16 minutes will result in noticeable cognitive and subjective manifestations. Maintaining that exertion for longer durations, for example approximately 24 minutes, will lead to heat casualties in approximately $3 \%$ of the population. Over 32 minutes of enduring $5 \mathrm{Kcal}$ per minute will lead to about $50 \%$ of those exposed suffering heat stroke and rendering them unavailable for further action.

Figure 2 demonstrates the relationships between activity types, ambient temperatures and the resulting TSI levels. In the example, the individual dons the firefighting suit and goes into a burning compartment that has a high ambient temperature. Note that the TSI estimate rises to unacceptable levels in about 33 minutes even with effective insulation. These data indicate that this individual should be required to proceed to a staging area and afforded an opportunity to recover.

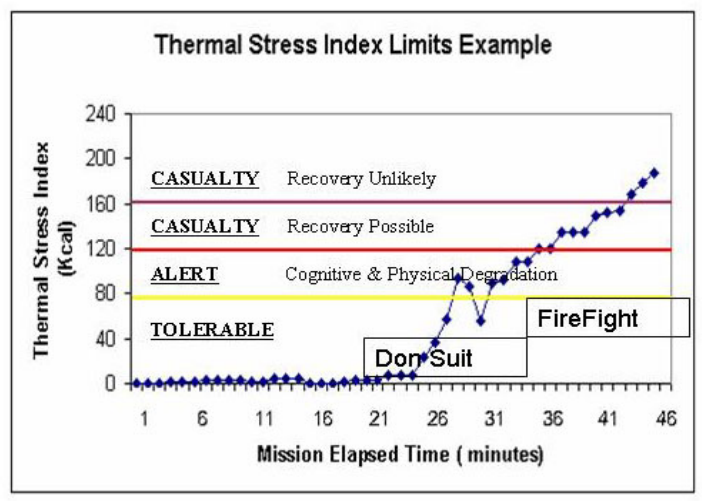

Figure 2. TSI Relationships.

Figure 3 is an example of damage control analysis using the TSI metric. In this example, the shipboard damage control team responses to two separate casualty events. The first casualty event is minor or acceptable on the bottom level in green on the chart and has a minimal impact on the thermal stress level experienced by damage control personnel. The second casualty event is more substantial stretching into the third highest or orange level of the chart and has a significant impact on the thermal stress level experienced by many of the damage control personnel. Note that two individual's peak at a TSI level of approximately 160 , which is in the unacceptable level.

High: possible immediate medical attention Very High: immediate required medical attention

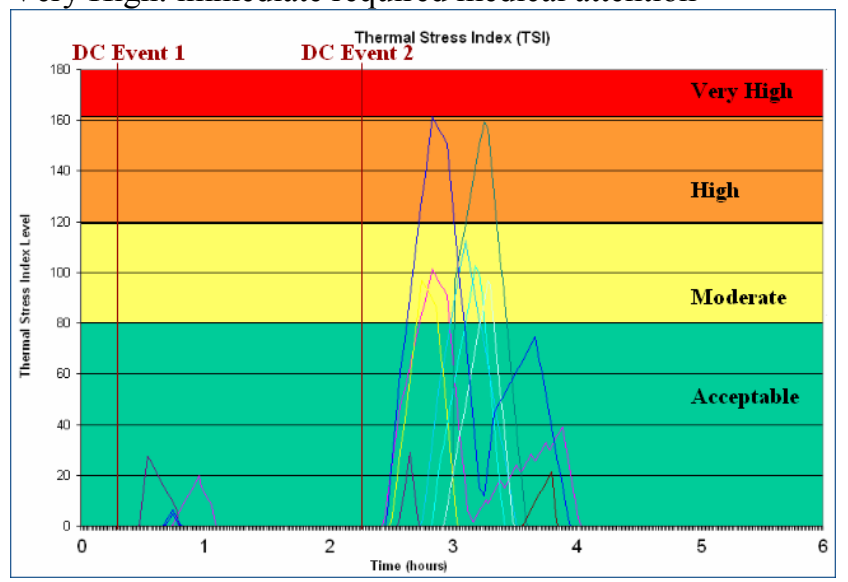

Figure 3. Example of TSI Analysis.

\section{FATIGUE}

Fatigue is a persistent threat to meeting mission requirements. A great deal is known about the effects of fatigue on cognitive ability as reliably demonstrated in sleep deprivation studies (Firoica, et al. 1968). The extensive literatures on fatigue effects describe increased variability in motivation, work efficiency and increases in lapses of vigilance (Dinges, et al. 1988). Thus, the ability of a crew to quickly and accurately respond to even simple tasks, the penchant to become lost in details, the reduced ability to solve complex problems, the loss of motivation to find a solution and the inability to assess the risks of a mission adequately are all symptoms of fatigue.

The introduction of the FADE algorithm into the TCM allows fatigue effects to be predicted and fatigue-reducing strategies tested before exposing real crews to the consequences of fatigue. FADE is based on data collected at the Air Force Research Laboratory using USAF and USN pilots as subjects during a 52-hour sleep deprivation study. The algorithm is based on one of the fatigue sensitive tasks used, the Maniken task of the Divided Attention task. This task was selected because it is a complex visual task; it required the subject to pay attention to a signal on the screen while performing one of two other tasks, thereby dividing 
their attention. It is an intellectually challenging test that has consistently proven sensitive to fatigue and other stressors in a number of experiments and is similar to the kinds of visual and cognitive performance demands placed on console operators.

The FADE equation predicts the decrement in response time that would be experienced in performing this task. This creates an output where the FADE fatigue values are directly interpretable as the percent decrement in response time, for example a FADE value of 6 would mean that an individual is predicted to be $6 \%$ slower at the Manikin task than at full rested (0) fatigue state. The FADE equation considers the awake degradation, sleep recovery, and daily circadian components. Figure 4 shows the fatigue estimates based on these components. A parabolic recovery function was needed since shortened sleep, as occurs during sleep fragmentation, does not provide adequate rest. Roehrs (et al. 1994) has shown that sleep fragmentation in the absence of sleep loss causes excessive daytime sleepiness independent of the sleep fragmentation schedule used.

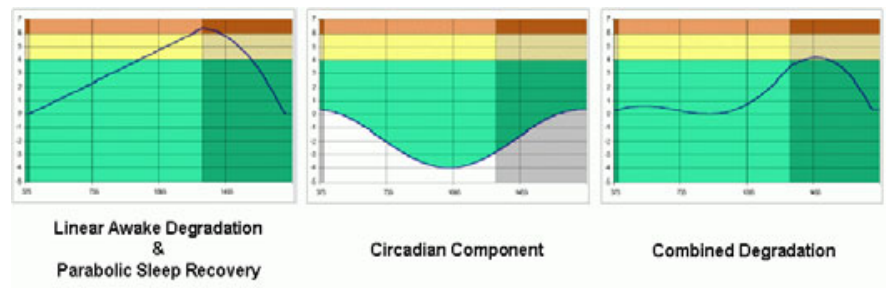

Figure 4. Combination of Linear/Parabolic and Circadian Component Effects on Fatigue

Figure 5 shows the FADE prediction for two different sleep/awake cycles. The fatigue associated with a normal sleep/awake cycle is shown in blue. The brown curve shows the fatigue levels during 48 hours of sleep deprivation. The oscillating circadian function is shown separately (in purple) to indicate the impact of circadian factors on fatigue. The arrows indicate limits set by us to guide the user in recommending levels of fatigue that would be considered excessive.

The area below the bottommost arrow (green) in Figure 5 borders where fatigue levels should be considered Acceptable. The Normal Rest curves, shown in blue, are the level of fatigue (up to 4 on the Fatigue Index score) that most people experience during their 16 hours awake assuming they be began the day well rested and are synchronous with their circadian cycle. Above the bottommost arrow (yellow) is an area that should be considered Marginally Acceptable. This is an area representing 16-18 hours of wakefulness and although humans can still function well, the average person is usually very tired at this point and in need of rest. Above the topmost arrow (red) starts an area of fatigue (Fatigue Index above 6) that repre-

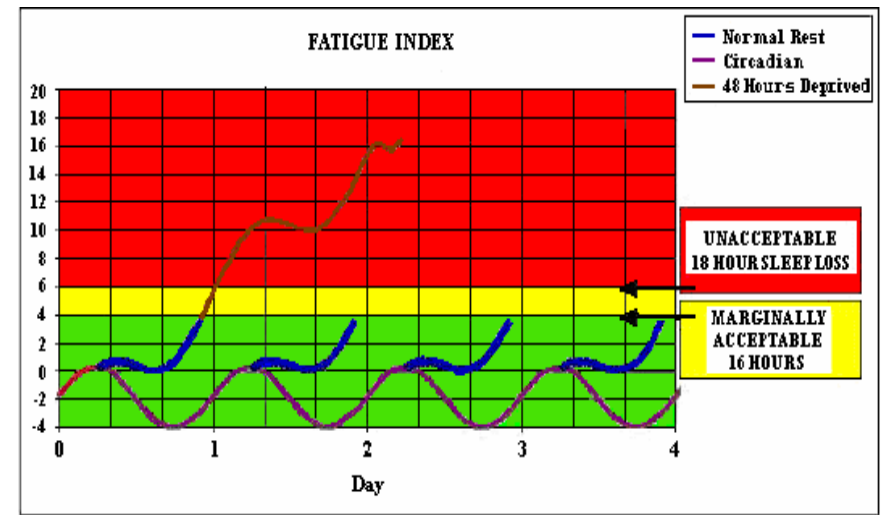

Figure 5. Fatigue Estimate for Various Sleep/Awake Cycles.

sents about 18 hours of wakefulness. This area is chosen as the beginning of the Unacceptable fatigue region due to the fact that beyond this level of fatigue, attentional lapses and microsleeps start to occur along with significant decrements in cognitive performance.

Figure 6 is an example of the application of the FADE equation in a TCM simulation. This figure demonstrates the estimated level of fatigue generated during the course of thirty-one days of sea duty for a select portion of the crew. It can be seen that, with this crew configuration, several Sonar Division crew members are often above the fatigue level identified as Unacceptable.

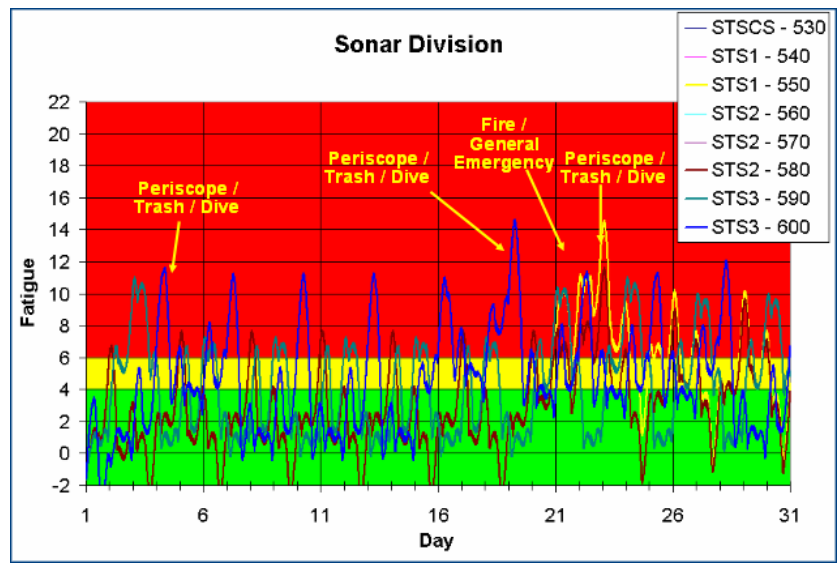

Figure 6. Example of FADE Analysis

It is obvious that humans are less alert and more prone to mistakes when their normal sleep is reduced or fragmented. The ability to predict when these impairments occur and to quantify the degree of impairment is a powerful tool. The FADE algorithm can produce empirical estimates of crew fatigue in the TCM model to assist in making command decisions relating to crew management. 


\section{PHYSIOLOGICAL STRESS INDEX}

Models of human performance frequently fail to account for the degradation resulting from physical human limitations such as from sleep loss or heat stress. The PSI attempts to increase the utility of modeling results by allowing computer simulations to estimate performance degradation based on the effects of thermal stress and fatigue. Therefore, the PSI can be used to improve the realism of simulation outcomes and can be used as a tool to rationally schedule safe exposure and recovery times for a variety of firefighting conditions.

The PSI combines the effects of TSI and FADE into one equation with the goal of providing a means for rapidly generating quantifiable physiological stress data for individuals or teams subject to the influences of physical activity, fatigue and physical environment. The two components of PSI (TSI and FADE) were normalized at the red line level for each. Specifically, the red line level for FADE (6) was normalized to make it equivalent to a red line level for TSI (160). After normalizing the two components they were summed together to generate the PSI value. Both FADE and TSI are assumed to have equivalent degrading effects on performance for a given level.

Figure 7 demonstrates the relationships between physiological stress levels and various combinations of fatigue levels and thermal stress levels.

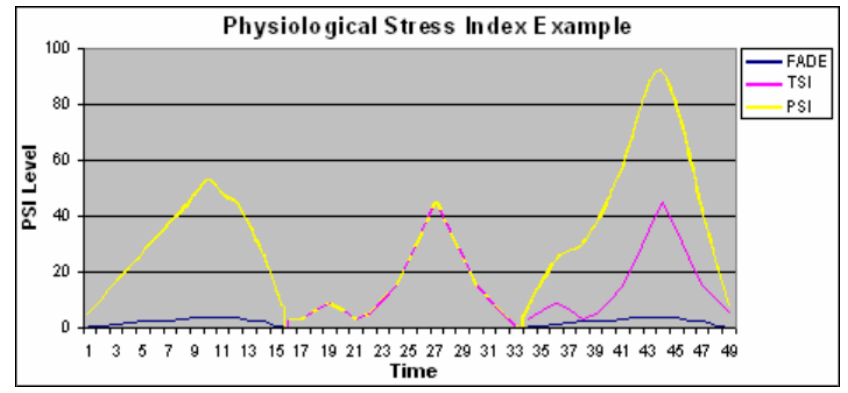

Figure 7. PSI Relationships.

Figure 8 is an example of PSI analysis data assuming that all crew members are fully rested $(\mathrm{FADE}=0)$ at the beginning of the six hour scenario. Note that the billet sequence numbers (BSN) with the highest PSI levels are 4, $30,33,57,61,63$ and 70 .

Figure 9 is an example of PSI analysis data with elevated crew fatigue levels at the beginning of the scenario. The starting fatigue levels for this data run are consistent with the ship having been underway for four weeks given a high tempo level of operations.

Note that the BSNs in Figure 9 with the highest PSI levels are 23, 34, 37, 46, 64 and 73 which are different from the BSNs identified in Figure 8, where all starting fatigue levels was zero.

The PSI is currently designed to estimate the consequences of individuals exposed to severe temperature

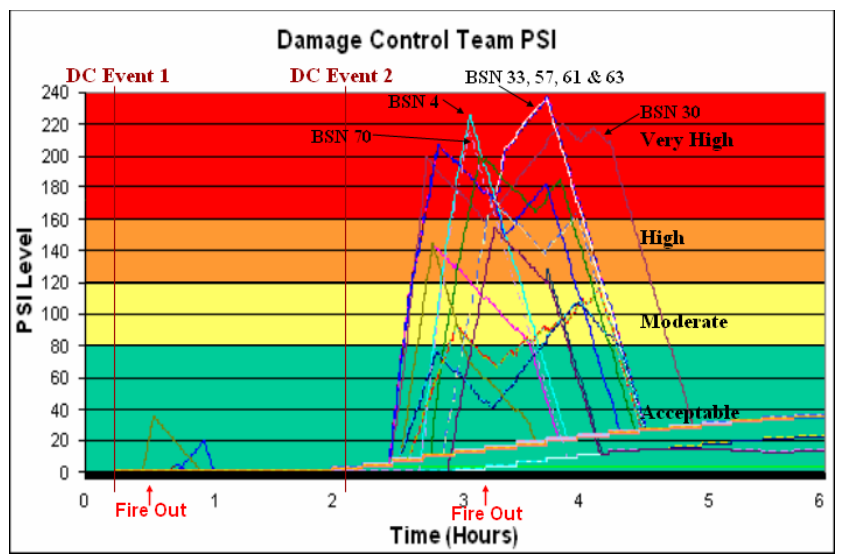

Figure 8. Example of PSI Analysis Assuming a Starting FADE Level of Zero

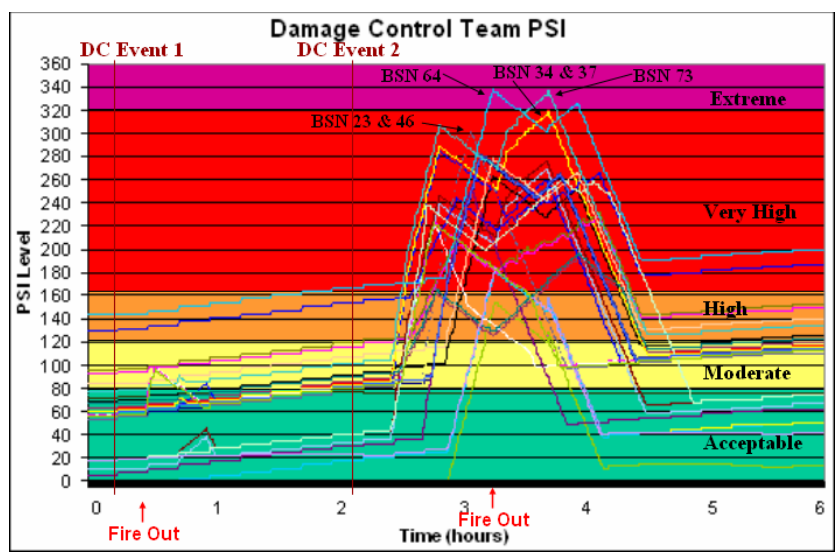

Figure 9. Example of PSI Analysis Assuming an Elevated Starting FADE Level

extremes while performing physically demanding tasks given various starting fatigue levels. In a discrete event simulation such as Micro Saint the PSI equation may be used to predict when specific crewmembers will become incapacitated and how long it will take for them to recover. Therefore this is a tool that may be used to evaluate a ship configuration to determine the optimum number of crewmembers required to operate a naval vessel during high tempo operations including casualty events. Additionally, this tool may be used to answer questions concerning the impact of losing key personnel as a result of combat losses or as a result of thermal stress casualties. The PSI may be used dynamically during the execution of a casualty scenario to control when damage control teams need to be relieved on station and to determine the time required before returning them to action.

It is programmatically convenient to combine a FADE score and a TSI score into a common psychological and physiological stress score, in this case the PSI. As the literature cited attests, there is also good reason to expect the cardiovascular, sympathetic and neuroendocrine respon- 
siveness, needed to confront the physically demanding requirements of onboard firefighting is weakened as a result of sleep deprivation. Eventually, we envision a family of stress effects to be incorporated into this generalized model of stress behavioral degradation that include, motion sensitivity, panic and other performance modifiers. For the time being, it is parsimonious to combine the two stressors and there is scientific support for this approach.

The limitations that human operators impose on task execution are rarely integrated into computer simulations of complex systems, resulting in considerable loss of outcome fidelity. Human behavior is limited by a number of frailties such as workload, exertion level, fatigue and thermal considerations. Using discrete-event simulation tools like Micro Saint, it is possible to stochastically model the impact of human interaction in system operations and to consider human capabilities thereby provide realistic outcome expectations for a variety of manning and mission scenarios (Laughery 1999). Task network simulation tools are particularly useful for military systems that can be costly to prototype and can require frequent design changes to accommodate advances in technology (Pew and Mavor 1997; See et al. 1997.) Verification and validation studies have been conducted favorably comparing Micro Saintbased simulation timing and workload predictions to real world military operations (McMahon, Spencer and Thornton 1995; Allender et al. 1997). The incorporation of PSI equations into Micro Saint simulations makes their predictions of human performance in complex operational environments far more realistic in terms of the impact of human limitations on model outcomes.

\section{REFERENCES}

Belding, H.S. 1973. Control of exposure to heat and cold. In Department of Health, Education, and Welfare, The industrial environment-Its evaluation and control. Washington, DC: U.S. Government Printing Office, 563-572.

Bowen, S., Wetteland, C. and French, J. 2003. The Total Crew Model (TCM): Using task network models to solve manning optimization issues. Third Annual Navy Workforce Research and Analysis Conference, March 31 - April 1, Alexandria, VA

Dinges, D.F., Whitehouse, W.G., Orne, E.C. and Orne, M.T. 1988. The benefits of a nap during prolonged work and wakefulness. Work and Stress, 2: 139-153.

Fiorica, V., Higgins, E.A., Iampietro, P.F., Lategola, M.T. and Davis. Q.W. 1968. Physiological responses of man during sleep deprivation. Journal of Applied Physiology 24 (2):169-175.

French, J., Miller, J., and Campbell, G. 2004. A model for estimating thermal stress and recovery cycles for firefighters. Proceedings of the Human Factors and Ergonomics Society Conference, , New Orleans, LA.
French, J., Morris, CS. and Hancock, PA. (2003) Modeling fatigue degraded performance in artificial agents, Proceedings of the Human Factors and Ergonomics Society, Denver, CO.

Hertig, B.A. Thermal standards and measurement techniques. 1973. In Department of Health, Education, and Welfare, The industrial environment - Its evalution and control. Washington, DC: U.S. Government Printing Office: 413-430.

Laughery, K.R. 1989. Micro Saint: A tool for modeling human performance in systems. In Applications of Human Performance Models to System Design, G.R. McMillan, D. Beevis, E. Salas, M.H. Strub, R. Sutton and L.van Breda (Eds) 219-230. New York, Plenum Press

Laughery, K.R. 1999. Modeling human performance during system design. In Human/Technology Interaction in Complex Systems, E. Salas (Ed) et al. 9: 147-174. Jai Press, Inc, Stamford, CT

McQuade, Allen J., U.S. Army Natick Development

\section{AUTHOR BIOGRAPHIES}

JON FRENCH is an associate professor of Human Factors and the Departmental Research Director at Embry Riddle Aeronautical University in Daytona Beach, FL. He has over 60 publications in the area of performance under stress. He was the Lead Behavioral Neuroscientist for Micro Analysis \& Design, Inc where he developed algorithms, such as fatigue, heat and motion sickness, to improve the realism of computer simulations of human performance. His e-mail address is frenc70feerau. edu.

JEFF MILLER is a staff analyst for Micro Analysis and Design, Inc. who is involved with various discrete task modeling projects designed to develop to workload and manning requirements for next generation combatant systems. Mr. Miller has extensive military operational experience obtained during his career as a Flight Officer in the United States Navy. Mr. Miller's educational experience includes a M.S. in Space Systems Operations through the Naval Post Graduate School, Monterey, CA (1994) and a B.S. in Electronic Engineering Technology through Florida A\&M, Tallahassee, FL His e-mail address is jmiller@maad. com. 